\title{
Variable Correlation between Bronchoalveolar Lavage Fluid Fungal Load and Serum-(1,3)- $\beta$-D-Glucan in Patients with Pneumocystosis-A Multicenter ECMM Excellence Center Study
}

\author{
Toine Mercier 1,2 ${ }^{(D)}$, Nesrine Aissaoui ${ }^{3}\left(\mathbb{D}\right.$, Maud Gits-Muselli $^{3}$, Samia Hamane ${ }^{3}$, \\ Juergen Prattes ${ }^{4} \mathbb{D}$, Harald H. Kessler ${ }^{5}$, Ivana Mareković ${ }^{6}$, Sanja Pleško ${ }^{6}$, Jörg Steinmann ${ }^{7,8}$, \\ Ulrike Scharmann ${ }^{8}(\mathbb{D})$, Johan Maertens ${ }^{1,2}(\mathbb{D})$, Katrien Lagrou ${ }^{1,9} \mathbb{D}$, Blandine Denis ${ }^{10}$, \\ Stéphane Bretagne ${ }^{3,11,12} \mathbb{D}$ and Alexandre Alanio ${ }^{3,11,12, * \mathbb{D}}$
}

1 Department of Microbiology, Immunology and Transplantation, KU Leuven, 3000 Leuven, Belgium; toine.mercier@uzleuven.be (T.M.); johan.maertens@uzleuven.be (J.M.); katrien.lagrou@uzleuven.be (K.L.)

2 Department of Hematology, University Hospitals Leuven, 3000 Leuven, Belgium

3 Laboratoire de Parasitologie-Mycologie, AP-HP, Groupe Hospitalier Saint-Louis-Lariboisière-Fernand-Widal, 75010 Paris, France; nesrine.aissaoui@aphp.fr (N.A.); maud.gits-muselli@aphp.fr (M.G.-M.); samia.hamane@aphp.fr (S.H.); stephane.bretagne@pasteur.fr (S.B.) Department of Internal Medicine, Section of Infectious Diseases and Tropical Medicine, Medical University of Graz, 8036 Graz, Austria; juergen.prattes@medunigraz.at

5 Diagnostic \& Research Institute of Hygiene, Microbiology and Environmental Medicine, Medical University of Graz, 8036 Graz, Austria; harald.kessler@medunigraz.at

6 Department of Clinical and Molecular Microbiology, University Hospital Centre Zagreb, School of Medicine, University of Zagreb, 10000 Zagreb, Croatia; imarekov@kbc-zagreb.hr (I.M.);

sanja.plesko@kbc-zagreb.hr (S.P.)

7 Institute for Clinical Hygiene, Medical Microbiology and Clinical Infectiology, Paracelsus Medical University, Nuremberg Hospital, 90419 Nuremberg, Germany; Joerg.Steinmann@klinikum-nuernberg.de

8 Institute of Medical Microbiology, University Hospital Essen, 45122 Essen, Germany;

Ulrike.Scharmann@uk-essen.de

9 Department of Laboratory Medicine and National Reference Center for Mycosis, University Hospitals Leuven, 3000 Leuven, Belgium

10 Service de Maladies Infectieuses et Tropicales, AP-HP, Groupe Hospitalier Saint-Louis-Lariboisière-Fernand-Widal, 75010 Paris, France; blandine.denis@aphp.fr

11 Department of Infectious Agents, Université de Paris, 75006 Paris, France

12 Molecular Mycology Unit, Centre National de la Recherche Scientifique (CNRS), Unité Mixte de Recherche UMR2000, Centre National de Référence Mycoses Invasives et Antifongiques (CNRMA), Institut Pasteur, 75724 Paris, France

* $\quad$ Correspondence: alexandre.alanio@pasteur.fr; Tel.: +33-140-61-3255; Fax: +33-145-68-8420

Received: 23 October 2020; Accepted: 26 November 2020; Published: 1 December 2020

\begin{abstract}
Pneumocystis jirovecii pneumonia is a difficult invasive infection to diagnose. Apart from microscopy of respiratory specimens, two diagnostic tests are increasingly used including real-time quantitative PCR (qPCR) of respiratory specimens, mainly in bronchoalveolar lavage fluids (BAL), and serum $\beta-1,3-\mathrm{D}$-glucan (BDG). It is still unclear how these two biomarkers can be used and interpreted in various patient populations. Here we analyzed retrospectively and multicentrically the correlation between BAL qPCR and serum BDG in various patient population, including mainly non-HIV patients. It appeared that a good correlation can be obtained in HIV patients and solid organ transplant recipients but no correlation can be observed in patients with hematologic malignancies, solid cancer, and systemic diseases. This observation reinforces recent data suggesting that BDG is not the best marker of PCP in non-HIV patients, with potential false positives due to other IFI or bacterial infections and false-negatives due to low fungal load and low BDG release.
\end{abstract}


Keywords: Pneumocystis jirovecii; qPCR; broncho-alveolar lavage fluid; fungal load; biomarker; $(1,3)$ - $\beta$-D-glucan; non-HIV patient

\section{Introduction}

Pneumocystis jirovecii pneumonia (PCP) is one of the most prevalent invasive fungal infections [1]. It is still one of the main infections revealing AIDS in western countries [2]. In parallel, it is now mainly diagnosed in non-HIV patients, such as patients treated for hematological malignancies, solid organ transplant recipients, or patients treated with immunosuppressive therapies [3]. The clinical presentation and biological features associated with PCP are different in HIV and non-HIV patients, suggesting that the disease has a different pathophysiology with a more acute disease presentation and an increased mortality in non-HIV patients [4].

The diagnosis of PCP has relied on microscopy with immunofluorescence as the most sensitive test to visualize the trophic forms and asci containing ascospores in respiratory specimens since the 1970s [5]. Since then, PCR for the sensitive detection of $P$. jirovecii DNA in respiratory specimens [6] and 1,3- $\beta$-D-glucan (BDG) assays for the detection of specific polysaccharides from the asci cell wall in serum [7]. were developed and evaluated in the 1990s. More recently, real-time quantitative PCR (qPCR) assays have been developed and are nowadays the only PCR method recommended for diagnosis [8]. Recommendations for diagnosis of PCP in patients with hematological malignancies rely on qPCR and BDG testing, with various flow-chart procedures depending on the type of specimen undergoing testing [8]. Evaluation of qPCR assays have shown excellent sensitivity and negative predictive values, allowing QPCR in bronchoalveolar lavage (BAL) fluid to be used to rule out the infection $[8,9]$. On the other hand, serum BDG initially showed excellent performance with $94.8 \%$ sensitivity and an area under the curve (AUC) of 0.965 [10]. However, recent studies, including another meta-analysis, tempered these results and highlighted the fact that the diagnostic accuracy of serum BDG in non-HIV patients seemed to be less sensitive [11,12]. The meta-analysis from Corpo et al. concluded that pooled sensitivity of BDG is thus insufficient to exclude PCP [11]. One strategy could therefore be to combine BDG in serum and qPCR in respiratory specimens to obtain more accurate results. When plotting BAL fungal load by qPCR and serum BDG titers taken within 15 days of bronchoscopy $(n=46)$, a weak but significant correlation between the log-transformed values was found with a regression line significantly different of $0(p=0.0005)$ and a slope of $0.33\left(R^{2}=0.2459\right)$ [13]. Another study based on cancer patient specimens also reported a weak correlation between both markers (Spearman index at 0.38) [12].

The aim of this study was to investigate the potential correlation between BAL qPCR fungal load and serum BDG in various populations of patients based on their underlying diseases.

\section{Materials and Methods}

\subsection{Participating Centers and Patients}

All Excellence Centers from the ECMM network (https://www.ecmm.info/ecmm-excellencecenters/) were invited to participate to the study and five centers accepted to include patients in this multicenter study. Centers were anonymized as Center 1 to Center 5 .

Patients were retrospectively enrolled between 1 January 2015 to 31 December 2019. Inclusion of the cases required that BAL fluid was tested with qPCR and a minimum of one serum for BDG was tested within a time frame of 7 days before or after BAL sampling. Coinfection with other invasive fungal infections were collected in parallel.

We anonymously collected the underlying disease and the final diagnosis, classified by the treating clinician as PCP or Pneumocystis carriage (PCC). PCP was defined as cases associated with a positive Pneumocystis jirovecii qPCR in BAL sample together with classical radiological signs (bilateral ground 
glass opacities on chest computed tomography or bilateral diffuse interstitial infiltrates on chest X-ray), and any of the following clinical signs and symptoms: dyspnea, cough, or hypoxemia.

\section{2. (1,3)- $\beta$-D-Glucan Assay}

BDG was performed in each center using the Fungitell assay (Associates of Cape Cod) in accordance with the manufacturer's instructions. BDG $>80 \mathrm{pg} / \mathrm{mL}$ was considered positive. BDG categories were defined as $<80,80-200,200-523$, and $>523 \mathrm{pg} / \mathrm{mL}$ to separate negative, weakly positive, positive, and strongly positive results. BDG $>523 \mathrm{pg} / \mathrm{mL}$ were arbitrarily set at $524 \mathrm{pg} / \mathrm{mL}$.

\section{3. $q P C R$ Assays and Calibration}

qPCR was performed in each center using various kits and assays (Supplemental Table S1). A calibrator specimen (pooled $P$. jirovecii-positive DNA in pooled P. jirovecii-negative DNA extracted from BAL as described earlier [14]) was sent to all participating centers and tested using the local routine qPCR assay. The quantification cycle $(\mathrm{Cq})$ value of the calibrator was then used to adjust all previously collected qPCR Cq values from all respective centers. Basically, the mean Cq value of the calibrator obtained from the five centers was calculated. An adjustment factor was calculated by subtracting the mean $\mathrm{Cq}$ value of the calibrator to the $\mathrm{Cq}$ value of the calibrator obtained by each center. The adjustment factor of each center was then subtracted from the Cq values of each BAL tested in that specific center in order to obtain the adjusted-Cq value.

\subsection{Graphs and Statistical Analysis}

Graph and statistical analyses were performed using Prism v. 8.4.3 (GraphPad Software). ANOVA Kruskal-Wallis tests were performed on categorical data and Mann-Whitney unpaired non-parametric tests were performed accordingly. $\mathrm{Cq}$ values and BDG titers $(\mathrm{pg} / \mathrm{mL})$ were used for $\mathrm{QPCR}$ and BDG data analysis. Medians and interquartile ranges are provided for data with non-gaussian distributions.

\section{Results}

A total of 147 patients were enrolled in this study, including 117 cases of Pneumocystis pneumonia (PCP) and 30 cases of Pneumocystis carriage (PCC). The distribution of the enrolment is shown in Table 1. Most of the patients were HIV-negative (91.2\%), including hematological malignancies $(46.3 \%)$, solid cancer $(17.7 \%)$, solid organ transplant $(8.8 \%)$, systemic diseases $(9.5 \%)$, and other underlying diseases (8.8\%) (Table 2). The median delay between serum sampling and BAL was 0 days [IQR 0-1].

The fungal load was significantly different between PCP and PCC patients with a median Cq of 28.0 [IQR 25.9-30.9] in PCP vs. 35.0 [IQR 33.5-36.8] in PCC patients $(p<0.0001)$ (Figure 1A). BDG was also significantly different between PCP and PCC patients with a median titer of $452 \mathrm{pg} / \mathrm{mL}$ [IQR 158-524] in PCP vs. 16.30 [IQR 7.70-79.25] $(p<0.0001)$ (Figure 1B). The AUC was 0.922 for qPCR in BAL, and 0.928 for serum BDG. The optimal threshold values to discriminate between PCP and PCC appeared to be $\mathrm{Cq}=34$ (sensitivity, 70\%; specificity, 95.7\%; positive likelihood ratio, 16.4) and BDG $=192 \mathrm{pg} / \mathrm{mL}$ (sensitivity, 100\%; specificity, 72.6\%; positive likelihood ratio, 3.6) (Figure 1C,D, Supplemental Tables S2 and S3).

Table 1. Distribution of enrolment in the five centers.

\begin{tabular}{cccc}
\hline & Patients Enrolled, $\boldsymbol{n} \mathbf{( \% )}$ & PCP, $\boldsymbol{n} \mathbf{( \% )}$ & PCC, $\boldsymbol{n} \mathbf{( \% )}$ \\
\hline Center 1 & $66(44.9)$ & $66(56.4)$ & 0 \\
Center 2 & $65(44.2)$ & $37(31.6)$ & $28(93.3)$ \\
Center 3 & $8(5.4)$ & $6(5.1)$ & $2(6.7)$ \\
Center 4 & $4(2.7)$ & $4(3.4)$ & 0 \\
Center 5 & $4(2.7)$ & $4(3.4)$ & 0 \\
Total & 147 & 117 & 30 \\
\hline
\end{tabular}


Table 2. Distribution of the underlying diseases.

\begin{tabular}{cccc}
\hline Underlying Diseases & All, $\boldsymbol{n} \mathbf{( \% )}$ & PCP, $\boldsymbol{n} \mathbf{( \% )}$ & PCC, $\boldsymbol{n} \mathbf{( \% )}$ \\
\hline Hematological malignancies & $68(46.3)$ & $49(41.9)$ & $19(63.3)$ \\
Solid Cancer & $26(17.7)$ & $24(20.5)$ & $2(6.7)$ \\
HIV & $13(8.8)$ & $11(9.4)$ & $2(6.7)$ \\
Solid organ transplantation & $13(8.8)$ & $11(9.4)$ & $2(6.7)$ \\
Systemic disease & $14(9.5)$ & $10(8.5)$ & $4(13.3)$ \\
Other & $13(8.8)$ & $12(10.3)$ & $1(3.3)$ \\
Total & 147 & 117 & 30 \\
\hline
\end{tabular}
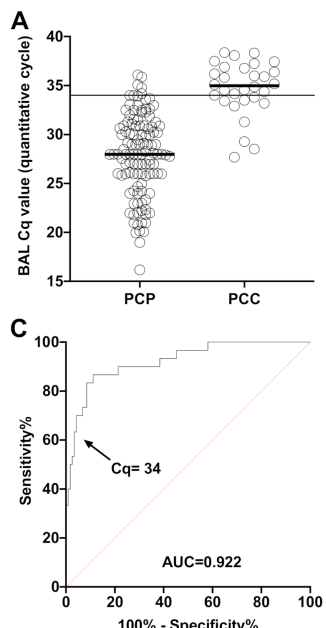

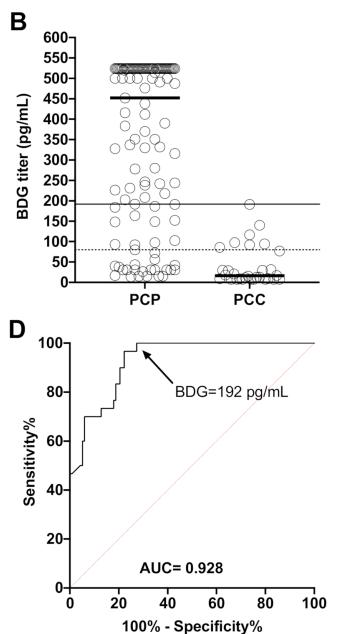

Figure 1. Distribution of the BAL Cq values (A) and BDG titers (B) and corresponding ROC curves for BAL Cq values (C) and BDG titers (D) comparing Pneumocystis pneumonia (PCP) and Pneumocystis carriage patients (PCC). Solid lines represent calculated optimal thresholds and black/red dotted lines the manufacturer threshold of the assay ( $80 \mathrm{pg} / \mathrm{mL}$ for Fungitell BDG assay).

In the full cohort, the regression line between fungal load (Cq value) and BDG titer was significantly different from 0 ( $p<0.0001)$ and showed a $R^{2}$ of 0.17 (Figure $\left.2 \mathrm{~A}\right)$. The distribution of the BAL fungal load was significantly different in the following BDG categories $<80,80-200,200-523,>523 \mathrm{pg} / \mathrm{mL}$ (Anova $p<0.0001$ ), with significantly lower Cq values in patients with $>523 \mathrm{pg} / \mathrm{mL}$ compared to other categories (Figure $2 \mathrm{~B}$ ).
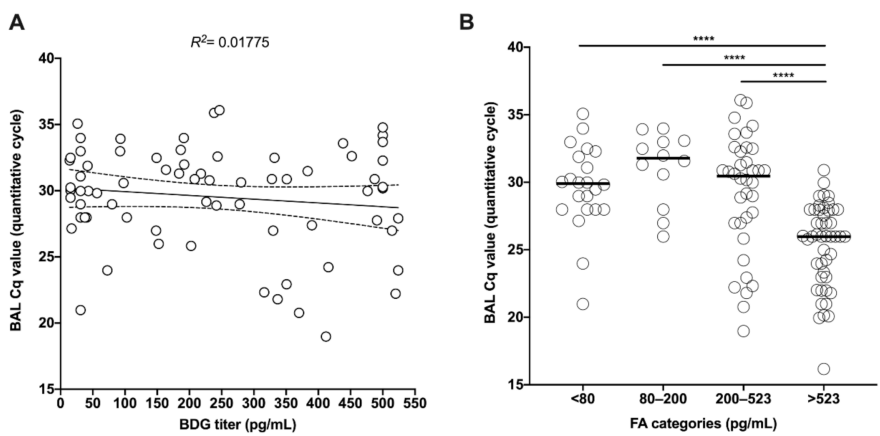

Figure 2. Linear (A) and categorial (B) correlation between BAL Cq value and BDG titer (pg/mL) in PCP patients. ${ }^{* * * *} p<0.0001$.

However, when focusing on various underlying diseases, some differences in the correlation appeared with the highest $R^{2}$ at 0.681 in HIV patients followed by solid organ transplant recipients $\left(R^{2}=0.573\right)$, systemic diseases $\left(R^{2}=0.326\right)$, and other underlying diseases $\left(R^{2}=0.179\right)$. A very weak 
correlation was observed in patients with hematological malignancies $\left(R^{2}=0.097\right)$ and solid cancer $\left(R^{2}=0.078\right)$ (Figure 3).
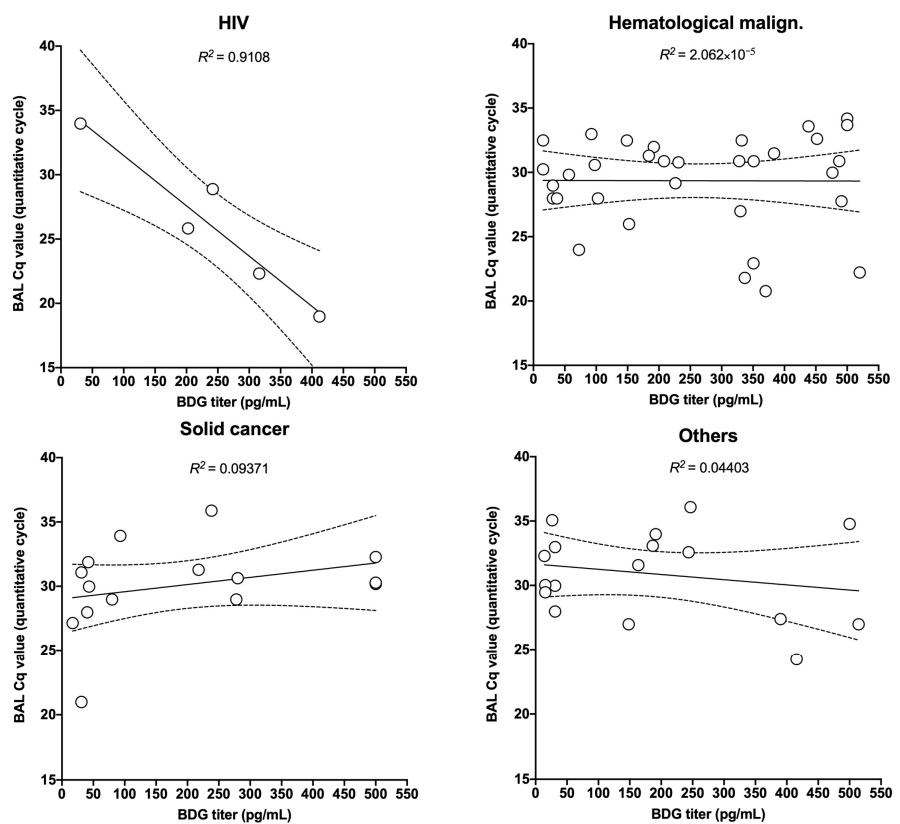

Figure 3. Linear correlation between BAL Cq value and BDG titer $(\mathrm{pg} / \mathrm{mL})$ according to the underlying diseases.

The distribution of the BAL Cq values was statistically different between the four BDG groups in hematological malignancies, solid cancer, solid organ transplant recipients, and others $(p<0.001)$. In all four groups, patients with BDG $>523$ had significantly lower $\mathrm{Cq}$ values than patients with lower BDG values (Figure 4). Of note, in HIV and systemic disease patients, Cq values were not significantly different between the different BDG categories.
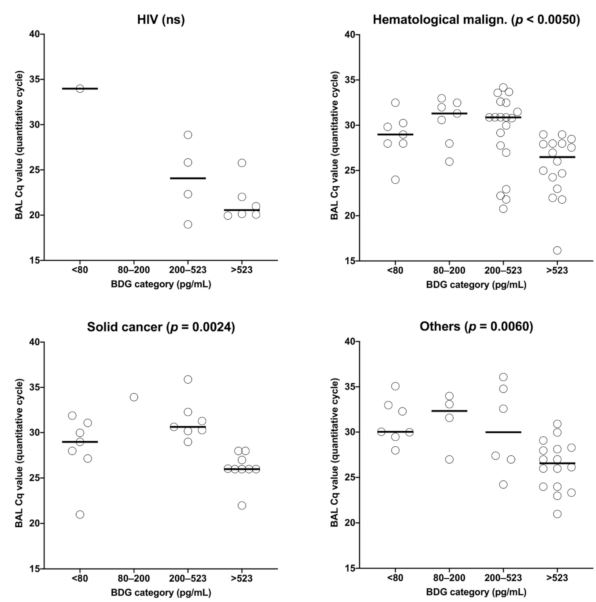

Figure 4. Categorial correlation between BAL Cq value and BDG titer $(\mathrm{pg} / \mathrm{mL})$ according to the underlying diseases.

\section{Discussion}

In this multicenter international retrospective study, we found a weak correlation between fungal load in BAL (as estimated by qPCR Cq values) and serum BDG value. Previous studies suggested that BDG in serum could be used as an estimator of the fungal burden, albeit with a weak correlation [12,13]. One possible explanation for the weak correlation could be the delay between BAL sampling and 
serum BDG testing, up to 15 days in one study [13], with no simultaneous increase or decrease, specifically after treatment, between the fungal load and the circulating antigen, as seen in Candida infections [15]. Indeed, we found a significant difference in fungal burden between patients having the highest BDG levels and the remainders. However, despite having most of the serum samples on the day of BAL sampling in this study, or the day before/after BAL sampling, we also found only a weak correlation with an $R^{2}$ of 0.17 for the whole cohort.

To analyze the correlation between qPCR and BDG, we faced the problem of heterogeneity of qPCR methods used in the five participating centers. This was not the case with BDG, since all participants used the Fungitell kit. Therefore, we circumvented this problem by using a qPCR calibrator sample sent to all participants. This allowed us to observe the differences in terms of quantification for a given sample in the various centers, as shown previously [14], and to calculate the adjusted qPCR result.

Since the diagnostic performance of BDG is different between HIV and non-HIV patients [11,12], we can presume a role for the immune system in the process of clearance from and/or release of BDG into the circulation. This is also evidenced by the differences in pathogenesis between these groups [4]. For this reason, we also stratified patients based on the underlying disease, and found a disease-dependent correlation between BDG and fungal load. In agreement with recent meta-analysis results, the strongest correlation was found in HIV-patients $\left(R^{2}=0.573\right)$, with the weakest correlation in solid cancer patients $\left(R^{2}=0.078\right)$ and hematologic malignancies $\left(R^{2}=0.097\right)$.

Other possible sources of increased variability-and thus a lower $R^{2}$ in cancer and hematology patients include concomitant invasive fungal infections (IFI) which could make BDG testing positive. In our study, only three non-HIV patients were reported to have a concomitant IFI (three invasive aspergilloses) together with PCP. One had a negative and two had positive ( $>500 \mathrm{pg} / \mathrm{mL})$ BDG tests. Indeed, this cannot explain why BDG and fungal load do not correlate well in non-HIV patients. One additional explanation could be the increased rate of concomitant bacterial infection which can lead to false positive BDG results [16-18]. In addition, the Fungitell assay is known to have significant analytical variability [19], which could impact the value of the BDG titer. On the other hand, the method of collection of BAL is known to be variable from a center to another and even from different wards (ICU vs. pneumology), allowing the introduction of variability in the quantification of the fungal load by qPCR [20]. Moreover, although there is a good correlation between Cq values and microscopic fungal burden (trophic or cystic forms per optical field) [21,22], this correlation is not perfect, adding another layer of variability [23].

It appears more and more clearly that in hematology, cancer, and systemic disease patients, BDG cannot rule out PCP. A positive BDG in this population should prompt a full microbiological work up to allow bacterial and fungal diagnosis outside PCP. This is why ECIL-5 experts placed BAL and Pneumocystis jirovecii detection as the first test to be performed in these patient populations [8] with BDG testing done as a second step. In contrast, BDG testing in addition to induced sputum for the detection of Pneumocystis seems to be a more suitable strategy to diagnose PCP in HIV patients. In non-HIV patients, Pneumocystis jirovecii qPCR plays a central role and is being standardized in order to implement it in clinical studies on the performance of a standard qPCR on the diagnosis of PCP.

Our study has several limitations. The study was not designed to evaluate the performance of BDG or qPCR, since the clinicians were aware of the results when they classified the patients. Indeed, some clinicians have integrated that some PCP can be BDG negative. It would have been interesting to know whether some BDG positive/qPCR negative patients were classified as PCP, but this will be investigated in the future.

We were able to enroll only a limited number of HIV-patients which prevents having strong evidence of the better correlation between BAL qPCR and serum BDG in HIV patients. However, comparing the results in HIV and in the other underlying diseases categories are very interesting and could give rise to specific studies in the future.

Another limitation is that, although we included samples from five expertise centers, the majority was included in two centers, which could result in confounding by a center effect. However, given that 
all parameters in this study are diagnostic parameters, any potential center-related differences in clinical management will not affect the (diagnostic) parameters used in this study. We therefore believe the potential bias to be small.

Additionally, the use of different qPCR methods between the different centers could be a potential source of variability. Although we did correct for this using a calibrator-based standardization method, some remaining variability cannot be excluded. Additionally, differences in BAL sampling such as the volume of liquid injected, can be a further source of variability. Moreover, the sample sizes of certain disease subgroups (such as HIV) were small, making statistical analysis difficult.

In conclusion, serum BDG values appear to offer some estimation of the fungal burden depending on the underlying disease (i.e., especially in HIV patients), but are not a perfect predictor of the qPCR results.

Supplementary Materials: The following are available online at http://www.mdpi.com/2309-608X/6/4/327/s1, Table S1: qPCR methodology in the five centers; Table S2: ROC curve sensibility and specificity data for BAL qPCR; Table S3: ROC curve sensibility and specificity data for BDG.

Author Contributions: Conceptualization, A.A.; Methodology, A.A., S.B., and T.M.; Validation, T.M. and A.A.; Formal analysis, A.A.; Resources, T.M., N.A., M.G.-M., S.H., H.H.K., S.P., U.S., and B.D.; Data curation, A.A.; Writing — original draft preparation, T.M. and A.A.; Writing—review and editing, T.M., J.P., I.M., J.S., J.M., K.L., B.D., S.B., and A.A.; Visualization, A.A; Supervision, A.A.; Project administration, A.A.; All authors have read and agreed to the published version of the manuscript.

Funding: This research received no external funding.

Acknowledgments: We would like to thank all the technical staff of the Mycology lab, Hôpital Saint-Louis, Paris France and in all the other Excellence Centers. We would like to thank the committee for evaluation of ECMM excellence centers: Cornelia Lass-Flörl; Austria (Microbiology Chair), Oliver Cornely; Germany (Clinical Chair); Alexandre Alanio (EC Study Coordinator); Jesus Guinea, Spain (Committee member); Orla Morrissey, Australia (Committee member); Alessandro Pasqualotto, Brazil (Committee member); Riina Richardson, UK (Committee member).

Conflicts of Interest: The authors declare no conflict of interest.

\section{References}

1. Bitar, D.; Lortholary, O.; Strat, Y.L.; Nicolau, J.; Coignard, B.; Tattevin, P.; Che, D.; Dromer, F. Population-Based Analysis of Invasive Fungal Infections, France, 2001-2010. Emerg. Infect. Dis. 2014, 20, 1163-1169. [CrossRef] [PubMed]

2. Thomas, C.F.; Limper, A.H. Current insights into the biology and pathogenesis of Pneumocystis pneumonia. Nat. Rev. Microbiol. 2007, 5, 298-308. [CrossRef] [PubMed]

3. Dunbar, A.; Schauwvlieghe, A.; Algoe, S.; van Hellemond, J.J.; Reynders, M.; Vandecasteele, S.; Boelens, J.; Depuydt, P.; Rijnders, B. Epidemiology of Pneumocystis jirovecii Pneumonia and (Non-)use of Prophylaxis. Front. Cell. Infect. Microbiol. 2020, 10, 224. [CrossRef] [PubMed]

4. Cordonnier, C.; Cesaro, S.; Maschmeyer, G.; Einsele, H.; Donnelly, J.P.; Alanio, A.; Hauser, P.M.; Lagrou, K.; Melchers, W.J.G.; Helweg-Larsen, J.; et al. Pneumocystis jirovecii pneumonia: Still a concern in patients with haematological malignancies and stem cell transplant recipients. J. Antimicrob. Chemother. 2016, 71, 2379-2385. [CrossRef]

5. Lim, S.K.; Eveland, W.C.; Porter, R.J. Direct Fluorescent-Antibody Method for the Diagnosis of Pneumocystis carinii Pneumonitis from Sputa or Tracheal Aspirates from Humans. Appl. Microbiol. 1974, 27, 144-149. [CrossRef]

6. Wakefield, A.E.; Pixley, F.J.; Banerji, S.; Sinclair, K.; Miller, R.F.; Moxon, E.R.; Hopkin, J.M. Detection of Pneumocystis carinii with DNA amplification. Lancet 1990, 336, 451-453. [CrossRef]

7. Yasuoka, A.; Tachikawa, N.; Shimada, K.; Kimura, S.; Oka, S. (1-) beta-D-glucan as a quantitative serological marker for Pneumocystis carinii pneumonia. Clin. Diagn. Lab. Immunol. 1996, 3, 197-199. [CrossRef]

8. Alanio, A.; Hauser, P.M.; Lagrou, K.; Melchers, W.J.G.; Helweg-Larsen, J.; Matos, O.; Cesaro, S.; Maschmeyer, G.; Einsele, H.; Donnelly, J.P.; et al. ECIL guidelines for the diagnosis of Pneumocystis jirovecii pneumonia in patients with haematological malignancies and stem cell transplant recipients. J. Antimicrob. Chemother. 2016, 71, 2386-2396. [CrossRef] 
9. Fan, L.-C.; Lu, H.-W.; Cheng, K.-B.; Li, H.-P.; Xu, J.-F. Evaluation of PCR in Bronchoalveolar Lavage Fluid for Diagnosis of Pneumocystis jirovecii Pneumonia: A Bivariate Meta-Analysis and Systematic Review. PLOS ONE 2013, 8, e73099. [CrossRef]

10. Karageorgopoulos, D.E.; Qu, J.-M.; Korbila, I.P.; Zhu, Y.-G.; Vasileiou, V.A.; Falagas, M.E. Accuracy of $\beta$-D-glucan for the diagnosis of Pneumocystis jirovecii pneumonia: A meta-analysis. Clin. Microbiol. Infect. 2013, 19, 39-49. [CrossRef]

11. Corpo, O.D.; Butler-Laporte, G.; Sheppard, D.C.; Cheng, M.P.; McDonald, E.G.; Lee, T.C. Diagnostic accuracy of serum (1-3)- $\beta$-D-Glucan for Pneumocystis jirovecii pneumonia: A systematic review and meta-analysis. Clin. Microbiol. Infect. 2020, 26, 1137-1143. [CrossRef] [PubMed]

12. Szvalb, A.D.; Malek, A.E.; Jiang, Y.; Bhatti, M.M.; Wurster, S.; Kontoyiannis, D.P. Serum (1,3)-Beta-D-Glucan has suboptimal performance for the diagnosis of Pneumocystis jirovecii pneumonia in cancer patients and correlates poorly with respiratory burden as measured by quantitative PCR. J. Infect. 2020, 81, 443-451. [CrossRef] [PubMed]

13. Damiani, C.; Gal, S.L.; Costa, C.D.; Virmaux, M.; Nevez, G.; Totet, A. Combined Quantification of Pulmonary Pneumocystis jirovecii DNA and Serum $(1 \rightarrow 3)$ - $\beta$-D-Glucan for Differential Diagnosis of Pneumocystis Pneumonia and Pneumocystis Colonization. J. Clin. Microbiol. 2013, 51, 3380-3388. [CrossRef] [PubMed]

14. Gits-Muselli, M.; White, P.L.; Mengoli, C.; Chen, S.; Crowley, B.; Dingemans, G.; Fréalle, E.; Gorton, R.L.; Guiver, M.; Hagen, F.; et al. The Fungal PCR Initiative's evaluation of in-house and commercial Pneumocystis jirovecii qPCR assays: Toward a standard for a diagnostics assay. Med. Mycol. 2019, 58, 779-788. [CrossRef]

15. Angebault, C.; Lanternier, F.; Dalle, F.; Schrimpf, C.; Roupie, A.-L.; Dupuis, A.; Agathine, A.; Scemla, A.; Paubelle, E.; Caillot, D.; et al. Prospective Evaluation of Serum $\beta$-Glucan Testing in Patients with Probable or Proven Fungal Diseases. Open Forum Infect. Dis. 2016, 3, ofw128. [CrossRef]

16. Azoulay, E.; Guigue, N.; Darmon, M.; Mokart, D.; Lemiale, V.; Kouatchet, A.; Mayaux, J.; Vincent, F.; Nyunga, M.; Bruneel, F.; et al. (1,3)- $\beta$-D-glucan assay for diagnosing invasive fungal infections in critically ill patients with hematological malignancies. Oncotarget 2016, 7, 21484. [CrossRef]

17. Marty, F.M.; Lowry, C.M.; Lempitski, S.J.; Kubiak, D.W.; Finkelman, M.A.; Baden, L.R. Reactivity of $(1 \rightarrow 3)-\beta$-D-Glucan Assay with Commonly Used Intravenous Antimicrobials. Antimicrob. Agents Chemother. 2006, 50, 3450-3453. [CrossRef]

18. Albert, O.; Toubas, D.; Strady, C.; Cousson, J.; Delmas, C.; Vernet, V.; Villena, I. Reactivity of (1 $\rightarrow 3)-\beta-D-$ glucan assay in bacterial bloodstream infections. Eur. J. Clin. Microbiol. 2011, 30, 1453-1460. [CrossRef]

19. Mercier, T.; Guldentops, E.; Patteet, S.; Beuselinck, K.; Lagrou, K.; Maertens, J. Beta-D-Glucan for Diagnosing Pneumocystis Pneumonia: A Direct Comparison between the Wako $\beta$-Glucan Assay and the Fungitell Assay. J. Clin. Microbiol. 2019, 57. [CrossRef]

20. Costabel, U. Bronchoalveolar lavage: A standardized procedure or a technical dilemma? Eur. Respir. J. 1991, 4, 776-777.

21. Alanio, A.; Desoubeaux, G.; Sarfati, C.; Hamane, S.; Bergeron, A.; Azoulay, E.; Molina, J.M.; Derouin, F.; Menotti, J. Real-time PCR assay-based strategy for differentiation between active Pneumocystis jirovecii pneumonia and colonization in immunocompromised patients. Clin. Microbiol. Infect. 2011, 17, 1531-1537. [CrossRef] [PubMed]

22. Mühlethaler, K.; Bögli-Stuber, K.; Wasmer, S.; von Garnier, C.; Dumont, P.; Rauch, A.; Mühlemann, K.; Garzoni, C. Quantitative PCR to diagnosePneumocystispneumonia in immunocompromised non-HIV patients. Eur. Respir. J. 2012, 39, 971-978. [CrossRef] [PubMed]

23. Bossart, S.; Mühlethaler, K.; Garzoni, C.; Furrer, H. Is real time PCR preferable to the direct immunofluorescence in the diagnosis of Pneumocystis jirovecii pneumonia in HIV-infected patients? BMC Res. Notes 2020, 13, 235. [CrossRef] [PubMed]

Publisher's Note: MDPI stays neutral with regard to jurisdictional claims in published maps and institutional affiliations. 